\title{
LHC as a photon-photon collider: Bounds on $\boldsymbol{\Gamma}_{X \rightarrow \gamma \gamma}$
}

\author{
S. I. Godunov $\odot,{ }^{1}$ E. K. Karkaryan $\odot,{ }^{2,3}$ V. A. Novikov $\odot,{ }^{1}$ A. N. Rozanov $\odot,{ }^{2,4}$ M. I. Vysotsky $\odot,{ }^{1}$ and E. V. Zhemchugov $\odot^{1,{ }^{*}}$ \\ ${ }^{1}$ I.E. Tamm Department of Theoretical Physics, Lebedev Physical Institute, 119991 Moscow, Russia \\ ${ }^{2}$ Institute for Theoretical and Experimental Physics, Moscow 117218 Russia \\ ${ }^{3}$ Moscow Institute of Physics and Technology (State University), Moscow 141701 Russia \\ ${ }^{4}$ Centre de Physique des Particules de Marseille, CPPM, Aix-Marseille Universite, \\ CNRS/IN2P3, Marseille F-13288, France
}

(Received 10 December 2020; accepted 8 January 2021; published 19 February 2021)

\begin{abstract}
In the relatively recent CMS data, there is a hint on the existence of a resonance with the mass $28 \mathrm{GeV}$ decaying to a $\mu^{+} \mu^{-}$pair and produced in association with a $b$ quark jet and a second jet. Such a resonance should also couple to photons through the fermion loop, therefore, it can be searched for in ultraperipheral collisions (UPC) of protons. We set an upper bound on the $X \gamma \gamma$ coupling constant from the data on $\mu^{+} \mu^{-}$ pair production in UPC at the LHC. Our approach can be used for similar resonances should they appear in the future.
\end{abstract}

DOI: 10.1103/PhysRevD.103.035016

\section{INTRODUCTION}

LHC designed as a proton-proton collider can also be considered as a photon-photon collider in which photons are produced in ultraperipheral collisions of protons. The interest in studying $\gamma \gamma$ collisions is twofold: first, QED processes like $\gamma \gamma \rightarrow l^{+} l^{-}[1-3], \gamma \gamma \rightarrow W^{+} W^{-}$[4-7], $\gamma \gamma \rightarrow$ $\gamma \gamma$ [8-10] are investigated at very high energies never before accessible at particle accelerators, and second, production of new exotic particles can be looked for. The case of long-lived heavy charged particles was considered in [11]. Dark matter particles are discussed in [12-14]. In the paper [15], the production of exclusive $\gamma \gamma \rightarrow \mu^{+} \mu^{-}$events in proton-proton collisions at a centerof-mass energy of $13 \mathrm{TeV}$ with the ATLAS detector was analyzed. The measurement was performed in the dimuon invariant mass interval $12 \mathrm{GeV}<m_{\mu^{+} \mu^{-}}<70 \mathrm{GeV}$. If a resonance with the mass in this interval does exist and can decay to a $\mu^{+} \mu^{-}$pair, we can obtain an upper bound on its coupling with two photons from the data provided in [15]. A hint of such a resonance $X$ with the mass $(28.3 \pm 0.4) \mathrm{GeV}$ was reported by the CMS Collaboration [16], and in what follows, we will obtain bounds on its coupling to two photons. However, being

\footnotetext{
*Corresponding author. evgenii.zhemchugov@cern.ch

Published by the American Physical Society under the terms of the Creative Commons Attribution 4.0 International license. Further distribution of this work must maintain attribution to the author(s) and the published article's title, journal citation, and DOI. Funded by SCOAP ${ }^{3}$.
}

universal, our approach can be used for another resonance if it exists. ${ }^{1}$

As it was noticed in [17], $X$ can be responsible for the deviation of the measured value of the muon anomalous magnetic moment $a_{\mu}$ from its theoretical value. Introducing the coupling $Y$ of the scalar $X$ to muons according to

$$
\Delta \mathcal{L}=Y \bar{\mu} \mu X
$$

it was obtained in [17] that for $Y=0.041 \pm 0.006$ one loop contribution $\delta a_{\mu}^{X}=(29 \pm 8) \times 10^{-10}$ explains the deviation of the measured value of $a_{\mu}$ from the Standard Model result. It was also shown that such couplings are consistent with other experimental bounds.

With this value of $Y$, we get

$\Gamma_{X \rightarrow \mu^{+} \mu^{-}}=\frac{Y^{2}}{8 \pi} M_{X}\left(1-\frac{4 m_{\mu}^{2}}{M_{X}^{2}}\right)^{3 / 2}=(1.8 \pm 0.5) \mathrm{MeV}$,

while according to [16], the width of the peak is

$$
\Gamma_{X}^{\exp }=(1.8 \pm 0.8) \mathrm{GeV},
$$

which is close to the detector mass resolution for a dimuon system $\sigma=0.45 \mathrm{GeV}$. That is why we will also consider the case of $\Gamma_{X}$ approximately equal to $\Gamma_{X \rightarrow \mu^{+} \mu^{-}}$ given in (2).

\footnotetext{
${ }^{1}$ In particular, the $X$ production mechanism in inclusive $p p$ collisions is not straightforward and requires introduction of other new particles [17]. This is not relevant for the $X$ production in $\gamma \gamma$ collision.
} 


\section{THE FIDUCIAL CROSS SECTION OF THE $p p(\gamma \gamma) \rightarrow p p \mu^{+} \mu^{-}$REACTION}

We are interested in the contribution of the $X$ resonance to this cross section. In [15], the cross section of $\mu^{+} \mu^{-}$ production was measured in four intervals of the muon pair invariant mass on which the entire interval $12 \mathrm{GeV}<$ $m_{\mu^{+} \mu^{-}}<70 \mathrm{GeV}$ was divided. We are interested in the interval $22 \mathrm{GeV}<m_{\mu^{+} \mu^{-}}<30 \mathrm{GeV}$, for which, according to Table 3 of [15],

$$
\begin{aligned}
\frac{d \sigma^{\exp }}{d m_{\mu^{+} \mu^{-}}}= & (0.076 \pm 0.005) \frac{\mathrm{pb}}{\mathrm{GeV}}, \\
& \text { hence } \sigma^{\exp }=(0.61 \pm 0.04) \mathrm{pb} .
\end{aligned}
$$

This cross section measurement corresponds to the fiducial region $p_{T}^{\mu}>\hat{p}_{T}=6 \mathrm{GeV}$ and $|\eta|<\hat{\eta}=2.4$, where $p_{T}^{\mu}$ is the component of the muon momentum transversal to the proton beam and $\eta$ is the muon pseudorapidity: $\eta=-\ln \tan (\theta / 2)$, where $\theta$ is the angle between the muon momentum and the beam. The ATLAS muon spectrometer is measuring muon momentum up to $|\eta|=2.7$, but the trigger chambers cover the range $|\eta|<2.4$ that corresponds to the pseudorapidity cutoff given above.

According to the equivalent photons approximation the cross section of $\mu^{+} \mu^{-}$pair production in ultraperipheral collisions is given by

$$
\begin{aligned}
& \sigma\left(p p(\gamma \gamma) \rightarrow p p \mu^{+} \mu^{-}\right) \\
& \quad=\int_{0}^{\infty} d \omega_{1} \int_{0}^{\infty} d \omega_{2} \sigma\left(\gamma \gamma \rightarrow \mu^{+} \mu^{-}\right) n\left(\omega_{1}\right) n\left(\omega_{2}\right),
\end{aligned}
$$

where $n(\omega)$ is the equivalent photons spectrum. In the leading logarithmic approximation (LL),

$$
n(\omega) \approx n_{\mathrm{LL}}(\omega)=\frac{2 \alpha}{\pi \omega} \ln \frac{\hat{q} \gamma}{\omega},
$$

where $\alpha$ is the fine structure constant, $\gamma=6.93 \times 10^{3}$ is the Lorentz factor of the proton with the energy $6.5 \mathrm{TeV}$, and $\hat{q}$ is the maximal photon momentum at which the proton does not disintegrate. In this approximation, the integrals in Eq. (5) are divergent, and the integration domain is cut off explicitly with $\hat{q} \gamma$,

$$
\begin{aligned}
& \sigma_{\mathrm{LL}}\left(p p(\gamma \gamma) \rightarrow p p \mu^{+} \mu^{-}\right) \\
& \quad=\int_{m_{\mu}^{2} / \hat{q} \gamma}^{\hat{q} \gamma} d \omega_{1} \int_{m_{\mu}^{2} / \omega_{1}}^{\hat{q} \gamma} d \omega_{2} \sigma\left(\gamma \gamma \rightarrow \mu^{+} \mu^{-}\right) n_{\mathrm{LL}}\left(\omega_{1}\right) n_{\mathrm{LL}}\left(\omega_{2}\right) .
\end{aligned}
$$

The value of $\hat{q}$ is determined by the proton form factor and numerically $\hat{q} \approx 0.20 \mathrm{GeV}$ [18].

It is convenient to substitute the integration over photon energies by integration over $s=4 \omega_{1} \omega_{2}$ and $x=\omega_{1} / \omega_{2}$.
Then Eq. (5) changes to

$$
\begin{aligned}
& \sigma\left(p p(\gamma \gamma) \rightarrow p p \mu^{+} \mu^{-}\right) \\
& \quad=\int_{\left(2 m_{\mu}\right)^{2}}^{\infty} \sigma\left(\gamma \gamma \rightarrow \mu^{+} \mu^{-}\right) d s \int_{0}^{\infty} \frac{d x}{8 x} n\left(\sqrt{\frac{s x}{4}}\right) n\left(\sqrt{\frac{s}{4 x}}\right) .
\end{aligned}
$$

To take the experimental cuts into account, we substitute $\sigma\left(\gamma \gamma \rightarrow \mu^{+} \mu^{-}\right)$by the differential over $p_{T}$ cross section,

$$
\begin{aligned}
& \sigma\left(p p(\gamma \gamma) \rightarrow p p \mu^{+} \mu^{-}\right) \\
& =\int_{\left(2 m_{\mu}\right)^{2}}^{\infty} d s \int_{0}^{\sqrt{s} / 2} \frac{d \sigma\left(\gamma \gamma \rightarrow \mu^{+} \mu^{-}\right)}{d p_{T}} d p_{T} \\
& \quad \times \int_{0}^{\infty} \frac{d x}{8 x} n\left(\sqrt{\frac{s x}{4}}\right) n\left(\sqrt{\frac{s}{4 x}}\right) .
\end{aligned}
$$

It is then straightforward to implement cuts over $s$ and $p_{T}$ by changing the integration limits to $\hat{s}_{\min }<s<\hat{s}_{\max }$ and $\hat{p}_{T}<p_{T}<\sqrt{s} / 2$ [assuming $\hat{s}_{\text {min }} \geq\left(2 \hat{p}_{T}\right)^{2} \gg\left(2 m_{\mu}\right)^{2}$ ]. To implement the cutoff over pseudorapidity, one should integrate over $x$ in the interval [18],

$\frac{1}{\hat{x}}<x<\hat{x}, \quad$ where $\hat{x}=\exp (2 \hat{\eta}) \frac{1-\sqrt{1-4 p_{T}^{2} / s}}{1+\sqrt{1-4 p_{T}^{2} / s}}$.

Let us note that in the leading logarithmic approximation from the condition $\omega \lesssim \hat{q} \gamma$, it follows that $x$ should be always smaller than $(2 \hat{q} \gamma / \sqrt{s})^{2}$. For numerical values of $\hat{\eta}$, $\hat{p}_{T}$, and $\hat{s}=\left\{\hat{s}_{\min }, \hat{s}_{\max }\right\}$ we are interested in, and for $x$ from the interval (10), this demand is satisfied.

Thus, for the fiducial cross section we obtain

$$
\begin{aligned}
\sigma_{\mathrm{fid}}^{\hat{s}, \hat{p}_{T}, \hat{\eta}}= & \int_{\hat{s}_{\min }}^{\hat{s}_{\max }} d s \int_{\hat{p}_{T}}^{\sqrt{s} / 2} \frac{d \sigma\left(\gamma \gamma \rightarrow \mu^{+} \mu^{-}\right)}{d p_{T}} d p_{T} \\
& \times \int_{1 / \hat{x}}^{\hat{x}} \frac{d x}{8 x} n\left(\sqrt{\frac{s x}{4}}\right) n\left(\sqrt{\frac{s}{4 x}}\right),
\end{aligned}
$$

where $\hat{x}$ is defined in (10). In the leading logarithmic approximation, the fiducial cross section is

$$
\begin{aligned}
\sigma_{\text {fid,LL }}^{\hat{s}, \hat{p}_{T}, \hat{\eta}}= & \frac{\alpha^{2}}{\pi^{2}} \int_{\hat{s}_{\min }}^{\hat{s}_{\max }} \ln ^{2} \frac{(2 \hat{q} \gamma)^{2}}{s} \frac{d s}{s} \int_{\hat{p}_{T}}^{\sqrt{s} / 2} \frac{d \sigma\left(\gamma \gamma \rightarrow \mu^{+} \mu^{-}\right)}{d p_{T}} \\
& \times\left[1-\frac{1}{3}\left(\frac{\ln \hat{x}}{\ln \frac{(2 \hat{q} \gamma)^{2}}{s}}\right)^{2}\right] \ln \hat{x} d p_{T} .
\end{aligned}
$$

Let us begin with the calculation of the Standard Model contribution to the cross section of $\mu^{+} \mu^{-}$pair production, given by the diagrams shown in Figs. 1(a) and 1(b).

The expression for the differential cross section is [[19], Chap. 88] 


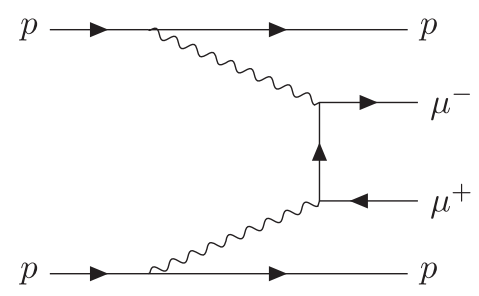

(a)

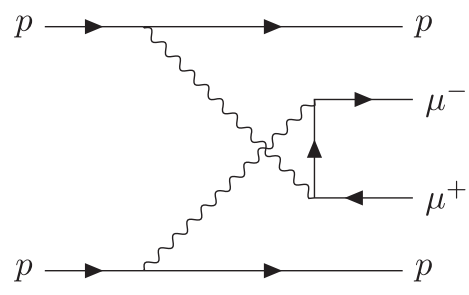

(b)

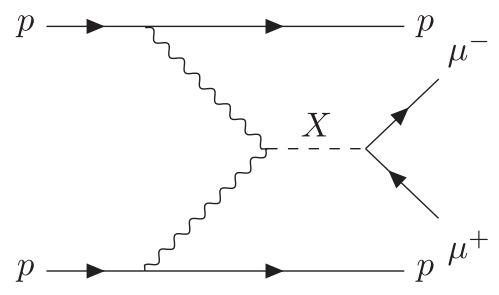

(c)

FIG. 1. Diagrams which contribute to the production of muon pair in ultraperipheral $p p$ collisions.

$$
\begin{aligned}
d \sigma\left(\gamma \gamma \rightarrow \mu^{+} \mu^{-}\right) & =\frac{2 \pi \alpha^{2}}{s^{2}}\left(\frac{s+t}{t}+\frac{t}{s+t}\right) d t \\
& =\frac{8 \pi \alpha^{2}}{s p_{T}} \frac{1-2 p_{T}^{2} / s}{\sqrt{1-4 p_{T}^{2} / s}} d p_{T}
\end{aligned}
$$

Substituting it in (12) and integrating over $p_{T}$, we get

$$
\begin{aligned}
\sigma_{\mathrm{fid}, \mathrm{sL}}^{\hat{s}, \hat{p}_{T}, \hat{\eta}} \approx & \frac{8 \alpha^{4}}{\pi} \int_{\hat{s}_{\min }}^{\hat{s}_{\max }} \ln ^{2} \frac{(2 \hat{q} \gamma)^{2}}{s} \frac{d s}{s^{2}} \\
& \times\left\{\hat{\eta}\left[\ln \left(\frac{1+\sqrt{1+4 \hat{p}_{T}^{2} / s}}{1-\sqrt{1-4 \hat{p}_{T}^{2} / s}}\right)-\sqrt{1-\frac{4 \hat{p}_{T}^{2}}{s}}\right]\right. \\
& -\frac{1}{4} \ln ^{2}\left(\frac{1+\sqrt{1+4 \hat{p}_{T}^{2} / s}}{1-\sqrt{1-4 \hat{p}_{T}^{2} / s}}\right) \\
& \left.+\frac{1}{2} \sqrt{1-\frac{4 \hat{p}_{T}^{2}}{s}} \ln \left(\frac{1+\sqrt{1+4 \hat{p}_{T}^{2} / s}}{1-\sqrt{1-4 \hat{p}_{T}^{2} / s}}\right)\right\} \\
= & 0.73 \mathrm{pb},
\end{aligned}
$$

where we neglected the small second term in the square brackets in (12) in order to perform integration analytically. Taking into account the omitted term and integrating numerically in (12), instead of $0.73 \mathrm{pb}$, we obtain $0.68 \mathrm{pb}$.

More accurate calculation depends on the internal structure of proton and the probability for the protons to survive the collision. For the latter, we will use the expression suggested in Ref. [20],

$$
P(b)=\left(1-\mathrm{e}^{-\frac{b^{2}}{2 B}}\right)^{2},
$$

where $b$ is the impact parameter of the collision, and $B$ was measured to be $19.7 \mathrm{GeV}^{-2}$ in the case of $p p$ collisions with the energy $7 \mathrm{TeV}$ [21]. To utilize this function, we introduce the equivalent photon spectrum at the distance $b$ from the source particle $n(b, \omega)$, such that

$$
n(\omega)=\int n(b, \omega) d^{2} b
$$

Then the leading logarithmic spectrum [[22], Chap. 15.5],

$$
n_{\mathrm{LL}}(b, \omega)=\frac{\alpha \omega}{\pi^{2} \gamma^{2}} K_{1}\left(\frac{b \omega}{\gamma}\right)
$$

where $K_{1}$ is the modified Bessel function of the second kind (the Macdonald function).

In the framework of the parton model and following Ref. [23], Eq. (5) is replaced with

$$
\begin{aligned}
& \sigma\left(p p(\gamma \gamma) \rightarrow p p \mu^{+} \mu^{-}\right) \\
& =\int_{0}^{\infty} d \omega_{1} \int_{0}^{\infty} d \omega_{2} \sigma\left(\gamma \gamma \rightarrow \mu^{+} \mu^{-}\right) \\
& \quad \times \int d^{2} b_{1} \int d^{2} b_{2} n\left(b_{1}, \omega_{1}\right) n\left(b_{2}, \omega_{2}\right) P\left(\left|\mathbf{b}_{1}-\mathbf{b}_{2}\right|\right) .
\end{aligned}
$$

This change is then propagated into Eq. (11),

$$
\begin{aligned}
\sigma_{\text {fid }}^{\hat{s}, \hat{p}_{T}, \hat{\eta}}= & \int_{\hat{s}_{\min }}^{\hat{s}_{\max }} d s \int_{\hat{p}_{T}}^{\sqrt{s} / 2} d p_{T} \frac{d \sigma\left(\gamma \gamma \rightarrow \mu^{+} \mu^{-}\right)}{d p_{T}} \\
& \times \int_{1 / \hat{x}}^{\hat{x}} \frac{d x}{8 x} \int_{b_{1}>0} d^{2} b_{1} \int_{b_{2}>0} d^{2} b_{2} n\left(b_{1}, \sqrt{\frac{s x}{4}}\right) \\
& \times n\left(b_{2}, \sqrt{\frac{s}{4 x}}\right) P\left(\left|\mathbf{b}_{1}-\mathbf{b}_{2}\right|\right) .
\end{aligned}
$$

The internal structure of proton is characterized by the Dirac form factor [24],

$$
\begin{aligned}
& F_{1}\left(Q^{2}\right)=G_{D}\left(Q^{2}\right)\left[1+\frac{\left(\mu_{p}-1\right) \tau}{1+\tau}\right], \\
& G_{D}\left(Q^{2}\right)=\frac{1}{\left(1+\frac{Q^{2}}{\Lambda^{2}}\right)^{2}},
\end{aligned}
$$

where $Q^{2}=-q^{2}, \quad q$ is the photon four momentum, $\tau=Q^{2} / 4 m_{p}^{2}, \quad m_{p}$ is the proton mass, and $\mu_{p}=$ 2.7928473508(85) is the proton magnetic moment [25], $G_{D}\left(Q^{2}\right)$ is the dipole form factor with $\Lambda$ being strictly fixed by the proton charge radius: $\Lambda^{2}=12 / r_{p}^{2}, r_{p}=$ $0.8751(61)$ fm [25].

The form factor enters Eqs. (5), (19) through the equivalent photon spectrum [18], 
TABLE I. The measured cross section for each interval of muon pair invariant mass and the corresponding theoretical calculations with different approximations: Eq. (12) is the calculation with the equivalent photon spectrum taken in the leading logarithmic approximation; Eqs. (11), (21) is the calculation taking into account the proton electromagnetic form factor; Eqs. (19), (22) also accounts for the probability of strong interactions at small impact parameters. "Survival ratio" is the ratio of the preceding two columns. Note that for the interval $30-70 \mathrm{GeV}$ the cutoff $\hat{p}_{T}=10 \mathrm{GeV}$ as it is in [15].

\begin{tabular}{lccccc}
\hline \hline & & $\begin{array}{c}\text { Leading logarithmic } \\
\text { approximation, Eq. (12) }\end{array}$ & $\begin{array}{c}\text { With the form factor, } \\
\text { Eqs. (11), (21) }\end{array}$ & $\begin{array}{c}\text { Also with the survival } \\
\text { factor, Eqs. (19), (22) }\end{array}$ & $\begin{array}{c}\text { Survival } \\
\text { ratio }\end{array}$ \\
\hline $12-17$ & $1.22 \pm 0.07$ & 1.25 & 1.28 & 1.24 & 0.970 \\
$17-22$ & $0.82 \pm 0.05$ & 0.87 & 0.896 & 0.866 & 0.967 \\
$22-30$ & $0.61 \pm 0.04$ & 0.68 & 0.703 & 0.677 & 0.963 \\
$30-70$ & $0.52 \pm 0.04$ & 0.49 & 0.506 & 0.483 & 0.953 \\
\hline \hline
\end{tabular}

$$
\begin{gathered}
n(\omega)=\frac{\alpha}{\pi^{2} \omega} \int \frac{\vec{q}_{\perp}^{2} F_{1}^{2}\left(\vec{q}_{\perp}^{2}+\omega^{2} / \gamma^{2}\right)}{\left(\vec{q}_{\perp}^{2}+\omega^{2} / \gamma^{2}\right)^{2}} \mathrm{~d}^{2} q_{\perp} \\
n(b, \omega)=\frac{\alpha}{\pi^{2} \omega}\left[\int \mathrm{d} q_{\perp} q_{\perp}^{2} \frac{F_{1}\left(q_{\perp}^{2}+\omega^{2} / \gamma^{2}\right)}{q_{\perp}^{2}+\omega^{2} / \gamma^{2}} J_{1}\left(b q_{\perp}\right)\right]^{2}
\end{gathered}
$$

where $\vec{q}_{\perp}$ is the photon transversal momentum, $J_{1}$ is the Bessel function of the first kind.

The so-called survival factor $S_{\gamma \gamma}^{2}[18,20,26]$ is defined as the ratio of the integrands in Eqs. (5), (18),

$$
S_{\gamma \gamma}^{2}=\frac{\int_{b_{1}>0} \int_{b_{2}>0} n\left(b_{1}, \omega_{1}\right) n\left(b_{2}, \omega_{2}\right) P\left(\left|\mathbf{b}_{\mathbf{1}}-\mathbf{b}_{\mathbf{2}}\right|\right) d^{2} b_{1} d^{2} b_{2}}{n\left(\omega_{1}\right) n\left(\omega_{2}\right)} ;
$$

however, in this paper, it is not calculated explicitly; Eq. (19) is used instead.

Calculations for each interval of muon pair invariant mass for Eq. (12), Eq. (11) with the spectrum (21), and Eq. (19) with the spectrum (22) are presented in the Table I. One can see that accounting for inelastic $p p$ scattering reduces the theoretical result by $5 \%$ approximately.

The amplitude of the $\mu^{+} \mu^{-}$pair production through intermediate $X$ boson in $\gamma \gamma$ collisions [see Fig. 1(c)] is given by the following expression:

$$
A=\kappa F_{\mu \nu}^{1} F_{\mu \nu}^{2} \frac{1}{s-M_{X}^{2}+i \Gamma_{X} M_{X}} \bar{\mu} \mu Y,
$$

\footnotetext{
${ }^{2}$ Survival factor can be also defined in a more elaborate way: on the amplitude level. See [27-33] for details. Let us also note that definition of $S_{\gamma \gamma}^{2}$ in Ref. [34] [Eq. (7)] is different: Ref. [34] requires that the new system is produced outside of the colliding particles, while Ref. [18] imposes no such restriction. The latter is more accurate when the new particles do not interact strongly, so we use the Ref. [18] definition of $S_{\gamma \gamma}^{2}$ here. In paper [33], it was specifically stressed that impact parameter cut like in Ref. [34] is unphysical.
}

where $\kappa$ is the $X \gamma \gamma$ coupling constant so that $\Gamma_{X \rightarrow \gamma \gamma}=$ $\left(\kappa^{2} M_{X}^{3}\right) /(16 \pi)$. For the cross section of the $\gamma \gamma \rightarrow X \rightarrow$ $\mu^{+} \mu^{-}$reaction, we obtain

$$
|A|^{2}=\kappa^{2} Y^{2} M_{X}^{6} \frac{1}{\left(s-M_{X}^{2}\right)^{2}+\Gamma_{X}^{2} M_{X}^{2}},
$$

$$
\sigma_{\gamma \gamma \rightarrow X \rightarrow \mu^{+} \mu^{-}}=\frac{2 \pi}{M_{X}^{2}} \frac{\Gamma_{X \rightarrow \gamma \gamma} \Gamma_{X \rightarrow \mu^{+} \mu^{-}}}{\left(\sqrt{s}-M_{X}\right)^{2}+\Gamma_{X}^{2} / 4},
$$

where the factor 2 takes into account identity of photons.

In the limit $m_{\mu} \rightarrow 0$ chiralities of the muons produced through the diagrams in Figs. 1(a), 1(b) are not the same as in Fig. 1(c). Consequently, these diagrams do not interfere in this limit. Even with nonzero $m_{\mu}$ the interference is zero at $s=M_{X}^{2}$ because then the phase between the sum of the diagrams in Figs. 1(a), 1(b), and the diagram in Fig. 1(c) is $\pi / 2$. For other values of $s$, the interference is suppressed relatively to $X$ contribution by the factor $\frac{\alpha \Gamma_{X}}{\sqrt{\Gamma_{X \rightarrow \mu^{+}}{ }^{-} \Gamma_{X \rightarrow \gamma \gamma}}} \frac{m_{\mu}}{M_{X}}\left(1-\frac{M_{X}^{2}}{s}\right)$, which is less than $10^{-2}$ for the largest allowed values of $\Gamma_{X \rightarrow \gamma \gamma}$ in both cases of the narrow or the wide resonance $\left(\Gamma_{X}=1.8 \mathrm{MeV}\right.$ or $1.8 \mathrm{GeV}$, respectively).

In order to impose the cut on the transverse momentum of muons with the help of expression (11) the following differential cross section is used:

$$
d \sigma=\frac{|A|^{2}}{32 \pi s} \frac{d\left(4 p_{T}^{2} / s\right)}{\sqrt{1-4 p_{T}^{2} / s}} .
$$

Substituting (27) and (25) in (12) and performing integration over $p_{T}$, we obtain 


$$
\begin{aligned}
& \sigma_{\mathrm{fid}, \mathrm{LL}}^{\hat{s}, \hat{p}_{T}, \hat{\eta}}(X) \\
& =\frac{8 \alpha^{2} \Gamma_{X \rightarrow \gamma \gamma} \Gamma_{X \rightarrow \mu^{+} \mu^{-}}}{\pi M_{X}^{2}} \int_{\hat{s}_{\min }}^{\hat{s}_{\max }} \frac{d s}{\left(s-M_{X}^{2}\right)^{2}+\Gamma_{X}^{2} M_{X}^{2}} \ln ^{2} \frac{(2 \hat{q} \gamma)^{2}}{s} \\
& \quad \times\left[\sqrt{1-\frac{4 \hat{p}_{T}^{2}}{s}}\left(2 \hat{\eta}+\ln \left(\frac{1-\sqrt{1-4 \hat{p}_{T}^{2} / s}}{1+\sqrt{1-4 \hat{p}_{T}^{2} / s}}\right)\right)-\ln \frac{4 \hat{p}_{T}^{2}}{s}\right] .
\end{aligned}
$$

In the case of a narrow resonance $\Gamma_{X} \approx \Gamma_{X \rightarrow \mu^{+} \mu^{-}}=$ $(1.8 \pm 0.5) \mathrm{MeV}$, the integration can be performed analytically, and we obtain

$$
\begin{aligned}
& \sigma_{\mathrm{fid}, \mathrm{LL}}^{\hat{s}, \hat{p}_{T}, \hat{\eta}}(X) \\
& =\frac{8 \alpha^{2} \Gamma_{X \rightarrow \gamma \gamma} \Gamma_{X \rightarrow \mu^{+} \mu^{-}}}{\Gamma_{X} M_{X}^{3}} \ln ^{2} \frac{(2 \hat{q} \gamma)^{2}}{M_{X}^{2}} \\
& \quad \times\left[\sqrt{1-\frac{4 \hat{p}_{T}^{2}}{M_{X}^{2}}}\left(2 \hat{\eta}+\ln \left(\frac{1-\sqrt{1-4 \hat{p}_{T}^{2} / M_{X}^{2}}}{1+\sqrt{1-4 \hat{p}_{T}^{2} / M_{X}^{2}}}\right)\right)-\ln \frac{4 \hat{p}_{T}^{2}}{M_{X}^{2}}\right] \\
& \approx 6.1 \times 10^{4} \frac{\Gamma_{X \rightarrow \mu^{+} \mu^{-}}}{M_{X}} \frac{\Gamma_{X \rightarrow \gamma \gamma}}{\Gamma_{X}} \mathrm{pb} .
\end{aligned}
$$

However, if $\Gamma_{X}=(1.8 \pm 0.8) \mathrm{GeV}$, then the width of the resonance almost equals $\sqrt{\hat{s}_{\max }}-M_{X}=2 \mathrm{GeV}$, so the integration should be done numerically, and we obtain

$$
\sigma_{\text {fid,LL }}^{\hat{s}, \hat{p}_{T}, \hat{\eta}}(X) \approx 49 \frac{\Gamma_{X \rightarrow \gamma \gamma}}{M_{X}} \mathrm{pb}
$$

\section{NUMERICAL ESTIMATES}

From the third line of the second and the fifth columns of the Table I, we see that the contribution of the resonance $X$ into the fiducial cross section of muon pair production is bounded in the following way ${ }^{3}$ :

$$
\sigma_{\text {fid }}(X) \lesssim 0.10 \mathrm{pb} \text { at } 99.5 \% \text { confidence level. }
$$

Comparing this number with the expression (29), we get that if $\Gamma_{X} \approx \Gamma_{X \rightarrow \mu^{+} \mu^{-}}$then the upper bound on $\Gamma_{X \rightarrow \gamma \gamma}$ is

$$
\begin{aligned}
\operatorname{Br}(X \rightarrow \gamma \gamma)< & 2.6 \times 10^{-2}, \\
\Gamma_{X \rightarrow \gamma \gamma}< & 46 \mathrm{keV} \approx 1.6 \times 10^{-6} M_{X} \text { at } 99.5 \% \\
& \text { confidence level }\left(\Gamma_{X}=1.8 \mathrm{MeV}\right) .
\end{aligned}
$$

If the width of $X$ is given by (3) then the bound extracted from Eq. (30) is

\footnotetext{
${ }^{3}$ When calculating the upper limit, in the case of negative signal, Ref. [35] suggests using zero instead of the negative value. This makes the upper limit a little less strong. This approach is widely used in the LHC experimental community, so we follow it here.
}

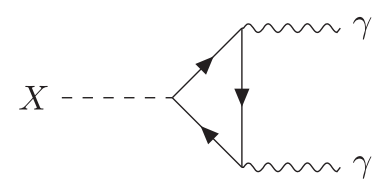

FIG. 2. Coupling of $X$ to two photons through a fermion loop.

$$
\begin{aligned}
\operatorname{Br}(X \rightarrow \gamma \gamma)< & 3.2 \times 10^{-2}, \\
\Gamma_{X \rightarrow \gamma \gamma}< & 58 \mathrm{MeV} \approx 2 \times 10^{-3} M_{X} \text { at } 99.5 \% \\
& \text { confidence level }\left(\Gamma_{X}=1.8 \mathrm{GeV}\right)
\end{aligned}
$$

Resonance $X$ couples with photons through a triangle diagram with fermion running in the loop (see Fig. 2). Let us check that the corresponding decay probability does not violate bounds just obtained.

The amplitude generated by the triangle diagram with a fermion $f$ running in the loop equals [36]

$$
A=\frac{\alpha F}{4 \pi} Y_{X f f} \frac{1}{m_{f}} X F_{\mu \nu}^{1} F_{\mu \nu}^{2} .
$$

The width equals

$$
\Gamma_{X \rightarrow \gamma \gamma}=\frac{\alpha^{2} F^{2}}{256 \pi^{3}} Y_{X f f}^{2}\left(\frac{M_{X}}{m_{f}}\right)^{2} M_{X},
$$

where

$$
\begin{gathered}
F=-2 \beta\left[(1-\beta) \varkappa^{2}+1\right], \quad \beta=\frac{4 m_{f}^{2}}{M_{X}^{2}}, \\
\varkappa=\left\{\begin{array}{l}
\arctan \left(\frac{1}{\sqrt{\beta-1}}\right), \beta>1 \\
\frac{1}{2}\left[i \ln \left(\frac{1+\sqrt{1-\beta}}{1-\sqrt{1-\beta}}\right)+\pi\right], \beta<1 .
\end{array}\right.
\end{gathered}
$$

For $m_{f} \ll M_{X}$, we obtain $F \sim\left(m_{f} / M_{X}\right)^{2}$, and for $m_{f} \gg M_{X}$, we obtain $F \rightarrow-4 / 3$.

In the case of muon running in the loop, we get $\Gamma_{X \rightarrow \gamma \gamma} \approx 10^{-11} M_{X}$, which is much smaller than bounds (32), (33). For a hypothetical fermion with a mass much larger than $M_{X}$, the width is also very small. However, for $m_{f} \sim M_{X}$ and $Y_{X f f} \sim 1$, it approaches $\mathrm{keV}: \Gamma_{X \rightarrow \gamma \gamma}\left(m_{f}=M_{X} / 2\right) \approx 3 Y_{X f f}^{2} \mathrm{keV}$.

\section{CONCLUSIONS}

A scalar resonance with the mass $28 \mathrm{GeV}$ coupling to muons in the way consistent with the recent CMS data [16] is also consistent with the measurements of the cross section for muon pair production in ultraperipheral 
collisions at the LHC [15], provided that the width of its decay to a pair of photons $\Gamma_{X \rightarrow \gamma \gamma}<46 \mathrm{keV}$ or $58 \mathrm{MeV}$ depending on whether the width $\Gamma_{X}=1.8 \mathrm{GeV}$ reported in Ref. [16] is the real width of the resonance or an artifact of the detector mass resolution.

The difference between the leading logarithmic approximation and the calculation that takes into account both the proton form factor and the survival factor for the protons colliding with the energy $13 \mathrm{TeV}$ is at the level of few percent. Integration of the logarithmic approximation can often be performed analytically while the form factor and especially the survival factor require computationally expensive numerical calculations. Therefore, cross sections for ultraperipheral collisions of protons in the lower region of invariant masses of the produced system can be estimated in the logarithmic approximation with the form factor and the survival factor taken into account as needed.

Our study demonstrates that we can look for New Physics in ultraperipheral collisions at the LHC.

\section{ACKNOWLEDGMENTS}

We are grateful to V. B. Gavrilov and A. N. Nikitenko, who have brought the CMS observation of $X(28 \mathrm{GeV})$ to our attention. We are grateful to V. A. Khoze for drawing our attention to papers [27-33]. We are supported by the Russian Science Foundation Grant No. 19-12-00123.
[1] CMS Collaboration, Exclusive photon-photon production of muon pairs in proton-proton collisions at $\sqrt{s}=7 \mathrm{TeV}$, J. High Energy Phys. 01 (2012) 052.

[2] ATLAS Collaboration, Measurement of exclusive $\gamma \gamma \rightarrow l^{+} l^{-}$ production in proton-proton collisions at $\sqrt{s}=7 \mathrm{TeV}$ with the ATLAS detector, Phys. Lett. B 749, 242 (2015).

[3] CMS Collaboration, Search for exclusive or semiexclusive photon pair production and observation of exclusive and semi-exclusive electron pair production in $\mathrm{pp}$ collisions at $\sqrt{s}=7 \mathrm{TeV}$, J. High Energy Phys. 11 (2012) 080 .

[4] CMS Collaboration, Study of exclusive two-photon production of $W^{+} W^{-}$in pp collisions at $\sqrt{s}=7 \mathrm{TeV}$ and constraints on anomalous quartic gauge couplings, J. High Energy Phys. 07 (2013) 116.

[5] CMS Collaboration, Evidence for exclusive $\gamma \gamma \rightarrow W^{+} W^{-}$ production and constraints on anomalous quartic gauge couplings in pp collisions at $\sqrt{s}=7$ and $8 \mathrm{TeV}$, J. High Energy Phys. 08 (2016) 119.

[6] ATLAS Collaboration, Measurement of exclusive $\gamma \gamma \rightarrow W^{+} W^{-}$production and search for exclusive Higgs boson production in pp collisions at $\sqrt{s}=8 \mathrm{TeV}$ using the ATLAS detector, Phys. Rev. D 94, 032011 (2016).

[7] ATLAS Collaboration, Observation of photon-induced $W^{+} W^{-}$production in pp collisions at $\sqrt{s}=13 \mathrm{TeV}$ using the ATLAS detector, CERN Report No. CERN-EP-2020165, 2020.

[8] ATLAS Collaboration, Evidence for light-by-light scattering in heavy-ion collisions with the ATLAS detector at the LHC, Nat. Phys. 13, 852 (2017).

[9] CMS Collaboration, Evidence for light-by-light scattering in ultraperipheral $\mathrm{PbPb}$ collisions at $\sqrt{s_{N N}}=5.02 \mathrm{TeV}$, Nucl. Phys. A982, 791 (2019).

[10] ATLAS Collaboration, Observation of Light-byLight Scattering in Ultraperipheral $\mathrm{Pb}+\mathrm{Pb}$ Collisions with the ATLAS Detector, Phys. Rev. Lett. 123, 052001 (2019).
[11] S. I. Godunov, V. A. Novikov, A. N. Rozanov, M. I. Vysotsky, and E. V. Zhemchugov, Quasistable charginos in ultraperipheral proton-proton collisions at the LHC, J. High Energy Phys. 01 (2020) 143.

[12] L. A. Harland-Lang, V. A. Khoze, M. G. Ryskin, and M. Tasevsky, LHC searches for dark matter in compressed mass scenarios: Challenges in the forward proton mode, J. High Energy Phys. 04 (2019) 010.

[13] V. A. Khoze, A. D. Martin, and M. G. Ryskin, Can invisible objects be 'seen' via forward proton detectors at the LHC? J. Phys. G 44, 055002 (2017).

[14] M. Tasevsky, L. A. Harland-Lang, V. A. Khoze, and M. G. Ryskin, Searches for dark matter at the LHC in forward proton mode, Proc. Sci., EPS-HEP2019 (2020) 492 [arXiv: 1910.01703].

[15] ATLAS Collaboration, Measurement of the exclusive $\gamma \gamma \rightarrow \mu^{+} \mu^{-}$process in proton-proton collisions at $\sqrt{s}=$ $13 \mathrm{TeV}$ with the ATLAS detector, Phys. Lett. B 777, 303 (2018).

[16] CMS Collaboration, Search for resonances in the mass spectrum of muon pairs produced in association with $b$ quark jets in proton-proton collisions at $\sqrt{s}=8$ and 13 TeV, J. High Energy Phys. 11 (2018) 161.

[17] S. I. Godunov, V. A. Novikov, M. I. Vysotsky, and E. V. Zhemchugov, Dimuon resonance near $28 \mathrm{GeV}$ and muon anomaly, JETP Lett. 109, 358 (2019).

[18] M. I. Vysotsky and E. V. Zhemchugov, Equivalent photons in proton-proton and ion-ion collisions at the LHC, Phys. Usp. 62, 910 (2019); Translated from Russian [UFN 189, 975 (2019)].

[19] V. B. Berestetskii, E. M. Lifshitz, and L. P. Pitaevskii, Quantum Electrodynamics (Pergamon Press, Oxford, 1982); Translated from Russian [Kvantovaya Elektrodinamika (Nauka, Moscow, 1989)].

[20] L. Frankfurt, C. E. Hyde-Wright, M. Strikman, and C. Weiss, Generalized parton distributions and rapidity gap survival in exclusive diffractive pp scattering, Phys. Rev. D 75, 054009 (2007). 
[21] ATLAS Collaboration, Measurement of the total cross section from elastic scattering in pp collisions at $\sqrt{s}=7 \mathrm{TeV}$ with the ATLAS detector, Nucl. Phys. B889, 486 (2014).

[22] J. D. Jackson, Classical Electrodynamics (John Wiley \& Sons, New York, 1962).

[23] R. N. Cahn and J. D. Jackson, Realistic equivalent-photon yields in heavy-ion collisions, Phys. Rev. D 42, 3690 (1990).

[24] S. Pacetti, R. B. Ferroli, and E. Tomasi-Gustafsson, Proton electromagnetic form factors: Basic notions, present achievements and future perspectives, Phys. Rep. 550-551, 1 (2015).

[25] P. J. Mohr, D. B. Newell, and B. N. Taylor, CODATA recommended values of the fundamental physical constants: 2014, Rev. Mod. Phys. 88, 035009 (2016).

[26] S. I. Godunov, V. A. Novikov, A. N. Rozanov, M. I. Vysotsky, and E. V. Zhemchugov (to be published).

[27] V. A. Khoze, A. D. Martin, R. Orava, and M. G. Ryskin, Luminosity measuring processes at the LHC, Eur. Phys. J. C 19, 313 (2001).

[28] V. A. Khoze, A. D. Martin, and M. G. Ryskin, Photon exchange processes at hadron colliders as a probe of the dynamics of diffraction, Eur. Phys. J. C 24, 459 (2002).

[29] V. A. Khoze, A. D. Martin, and M. G. Ryskin, Elastic scattering and diffractive dissociation in the light of LHC data, Int. J. Mod. Phys. A 30, 1542004 (2015).
[30] L. A. Harland-Lang, V. A. Khoze, M. G. Ryskin, and W. J. Stirling, Central exclusive production within the Durham model: A review, Int. J. Mod. Phys. A 29, 1430031 (2014).

[31] L. A. Harland-Lang, V. A. Khoze, and M. G. Ryskin, Exclusive physics at the LHC with SuperChic 2, Eur. Phys. J. C 76, 9 (2016).

[32] V. A. Khoze, A. D. Martin, and M. G. Ryskin, Multiple interactions and rapidity gap survival, J. Phys. G 45, 053002 (2018).

[33] L. A. Harland-Lang, M. Tasevsky, V. A. Khoze, and M. G. Ryskin, A new approach to modelling elastic and inelastic photon-initiated production at the LHC: SuperChic 4, Eur. Phys. J. C 80, 925 (2020).

[34] M. Dyndal and L. Schoeffel, The role of finite-size effects on the spectrum of equivalent photons in proton-proton collisions at the LHC, Phys. Lett. B 741, 66 (2015).

[35] G. Cowan, K. Cranmer, E. Gross, and O. Vitells, Asymptotic formulae for likelihood-based tests of new physics, Eur. Phys. J. C 71, 1554 (2011); Erratum, Eur. Phys. J. C 73, 2501 (2013).

[36] M. A. Shifman, A. I. Vainshtein, M. B. Voloshin, and V. I. Zakharov, Low-energy theorems for Higgs boson couplings to photons, Yad. Fiz. 30, 1368 (1979) [Sov. J. Nucl. Phys. 30, 711 (1979)], https://inspirehep .net/literature/141287. 